EPJ Web of Conferences 59, 12001 (2013)

DOI: $10.1051 /$ epjconf/20135912001

(C) Owned by the authors, published by EDP Sciences, 2013

\title{
Advances in target design and fabrication for experiments on NIF
}

\author{
K. Obrey ${ }^{1, a}$, D. Schmidt ${ }^{1}$, C. Hamilton ${ }^{1}$, D. Capelli ${ }^{1}$, J. Williams ${ }^{1}$, \\ R. Randolph ${ }^{1}$, F. Fierro ${ }^{1}$, D. Hatch ${ }^{1}$, G. Havrilla ${ }^{2}$ and B. Patterson ${ }^{1}$ \\ ${ }^{1}$ Los Alamos National Laboratory, Materials Science and Technology Division, Los Alamos, \\ NM 87544, USA \\ ${ }^{2}$ Los Alamos National Laboratory, Chemistry Division, Los Alamos, NM 87544, USA
}

\begin{abstract}
The ability to build target platforms for National Ignition Facility (NIF) is a key feature in LANL's (Los Alamos National Laboratory) Target Fabrication Program. We recently built and manufactured the first LANL targets to be fielded on NIF in March 2011. Experiments on NIF require precision component manufacturing and accurate knowledge of the materials used in the targets. The characterization of foams and aerogels, the Be ignition capsule, and machining unique components are of main material focus. One important characterization metric the physics' have determined is that the knowledge of density gradients in foams is important. We are making strides in not only locating these density gradients in aerogels and foams as a result of how they are manufactured and machined but also quantifying the density within the foam using 3D confocal micro $\mathrm{x}$-ray fluorescence $(\mu \mathrm{XRF})$ imaging and 3D x-ray computed tomography $(\mathrm{CT})$ imaging. In addition, collaborative efforts between General Atomics (GA) and LANL in the characterization of the NIF Ignition beryllium capsule have shown that the copper in the capsule migrates radially from the capsule center.
\end{abstract}

\section{ELEMENTAL DIFFUSION THROUGH IGNITION CAPSULES}

Recent collaborations with LANL and General Atomics (GA) have focused on analysis of ignition capsules, specifically elemental migration throughout the ignition capsules. Currently, the leading choice in the point design for the ignition capsules are graded doped $\mathrm{Cu}$-Be capsules with the secondary design being $\mathrm{Ge}$ doped $\mathrm{CH}$ capsules. Both of these capsule types were analyzed using 3D confocal $\mu$ XRF imaging and 3D x-ray CT imaging for possible elemental migration.

\subsection{Analysis of copper migration in graded doped $\mathrm{Cu}$-Be ignition capsules}

Previous studies at GA using contact radiography indicated migration of the $\mathrm{Cu}$ dopant in the capsule shell. In addition, SEM (scanning electron microscopy) images of pre- and post-pyrolysis indicates formation of domes on the surface of the capsules post-pyrolysis. We believe this is due to thermal migration of copper during the pyrolysis step. It is well known and documented in materials science literature that copper readily migrates when subjected to higher thermal conditions. [1] Since the "dome" features are seen on the capsule surface after pyrolysis, we believe these domes are that of copper migrating to the surface. To determine if the $\mathrm{Cu}$ migration occurred radially throughout the capsule, a few ignition capsules were given to LANL to study. Using a combination of 3D x-ray CT imaging and

\footnotetext{
${ }^{\mathrm{a}}$ e-mail: defriend@lanl.gov
}

This is an Open Access article distributed under the terms of the Creative Commons Attribution License 2.0, which permits unrestricted use, distribution, and reproduction in any medium, provided the original work is properly cited. 


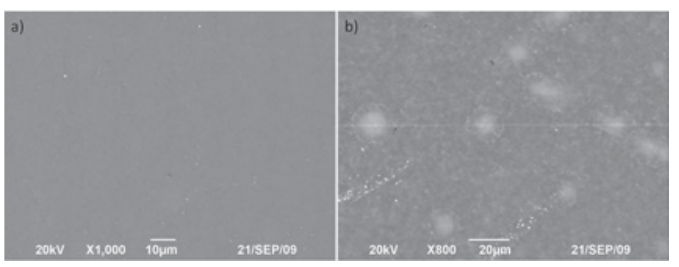

Figure 1. A sputtered Be-Cu capsule showing a) no BSE contrast from polished pre-pyro surface; b) lots of contrast from smooth surface on post-pyro surface.
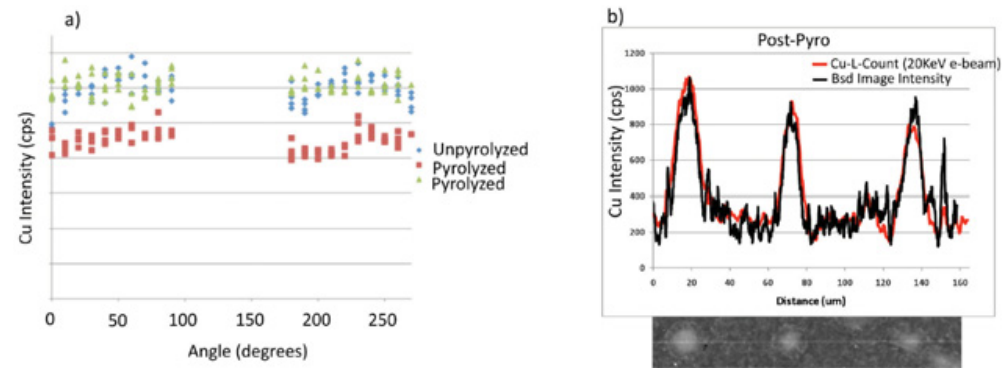

Figure 2. a) Averaged maximum $\mathrm{Cu}$ intensity as a function of theta showing variations in $\mathrm{Cu}$ amounts around the circumference of each capsule as well as variation between capsules, b) back-scattered electron (BSE) image of post-pyro outer surface shows line scan location and formation of copper islands matches to that on the capsule surface, courtesy of John Edwards, LLNL, and General Atomics.

3D confocal $\mu \mathrm{XRF}$ analyses, LANL determined that within the same batch, $\mathrm{Cu}$ migration was occurring within the shell and is not uniform from capsule-to-capsule $[2,3]$.

Some capsules within the same batch showed higher levels of copper migration than others, Fig. 2(a). The $\mathrm{Cu}$ migration is occurring from the higher concentration $\mathrm{Cu}$-doped inner layers out towards the outer surface where is there is no copper doping. This migration is a result of the heating process used in the removal of the plastic mandrel used in the sputtering process. This molten plastic passing through the shell wall is carrying some of the $\mathrm{Cu}$ with it. This results in the formation of streaks of copper as seen in the cross sectional images as well as copper islands forming on the surface, as seen in SEM images provided by LLNL and GA, Fig. 2(b). The graded Cu-doped Be capsules showed a higher amount of elemental migration compared to the second ignition capsule design, $\mathrm{Ge}-\mathrm{doped} \mathrm{CH}$ capsules, as explained below.

\subsection{Analysis of germanium migration in Ge-CH ignition capsules}

LANL applied the same techniques used for the $\mathrm{Cu}$-Be capsule analyses to the analysis $\mathrm{Ge}-\mathrm{CH}$ capsules. From these analyses, the Ge did not appear to be migrating in the capsules. GA provided LANL a series of capsules manufactured within the same batch, and all capsules indicated the placement and distribution of $\mathrm{Ge}$ to be relatively the same between each capsule, Fig. 3. These capsules showed a high amount of uniformity with a measurement noise between the capsules almost identical the measurement noise within the capsule. Note: There is a small amount of signal wobble seen in Figure 3 due to subresolution sample alignment off set in the instrument.

\section{PLEIADES TARGET FABRICATION AND ASSEMBLY}

As NIF comes on line and ignition becomes ever closer, LANL will be fielding more experiments on NIF. In March 2011, LANL fielded the first completely external experiment on NIF. The experiment 
IFSA 2011

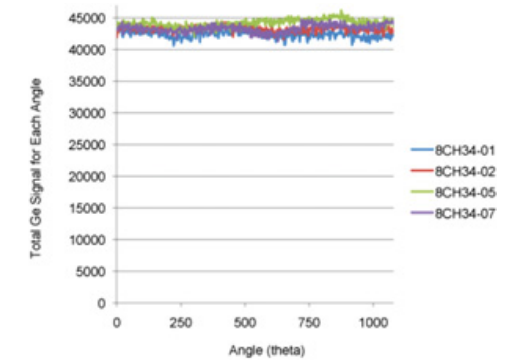

Figure 3. Summed Ge signal for each line scan, every 3 degrees for three runs around the circumference. There does not appear to be any variation in Ge signal between capsules.

a)

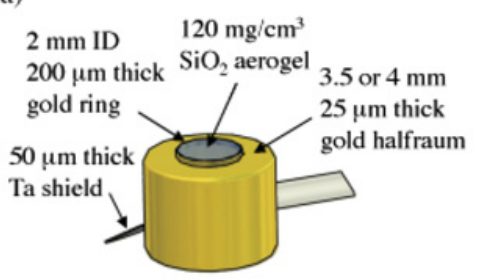

b)

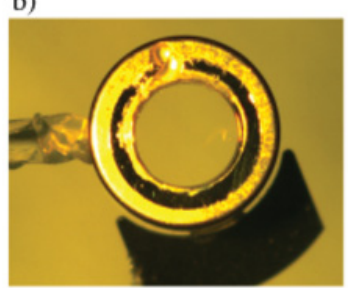

c)

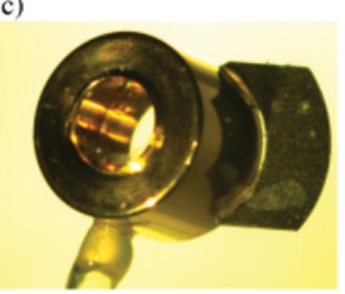

Figure 4. a) Illustration of the Pleiades Phase 1 target design, b) and c) photos of the final target assembly.

was a part of the Phase 1 in the Pleiades series. Since then, LANL has fielded three more experiments on NIF, also of the Pleiades target designs. Phase 1 of the Pleiades target consists of a thin ring filled with silica aerogel, which is mounted on top of a gold halfraum, Figure 4. Future phases will consist of a concentric cylinder design, where an outer silica aerogel tube is either filled with low-density foam or another density of silica aerogel. Assembly of the concentric cylinder target will take place on a robotic assembly station equipped with vacuum chucks to hold the delicate aerogel and foam pieces while insertion is occurring.

\section{ANALYSIS OF AEROGEL AND FOAMS USED IN PLEIADES TARGETS}

Analysis of silica aerogels and foams has shown that density gradients are present along the outer surface of the aerogel regardless of synthetic and processing methods used [4]. Typically, silica aerogel is processed by casting the alcogel inside a mold. After super critical extraction of the solvent, the resulting solid is no longer a gel, but is now an aerogel [5]. The gel may be cast into various shapes and sizes. Depending upon the mold used, the resultant aerogel matches to the shape of the mold. To easily remove the bulk piece of aerogel, a release agent is applied along the inside surface of the aerogel. The release agent is a fumed silica grease. Analysis of both aerogel with and without the release agent showed higher density formation along the surface, with the use of the releasing agent to a higher degree. After the bulk piece is removed from the mold, the aerogel can be machined, either by diamond turning or laser machining. Both processes have also shown the formation of density gradients. In essence, a higher density "skin" forms along the surface of the aerogel regardless of the processing and synthetic methods used [6].

LANL has found higher density forms at the surface of the aerogels when the aerogel is either cast or machined [6], Figure 5. Figure 5(a) shows a cast aerogel with no release agent, Fig. 5(b), a cast aerogel with release agent, and Fig. 5(c) an aerogel that was diamond turned. A drop in X-ray intensity is expected due to the geometry of the measurement, but comparing the amount of x-ray scatter in 

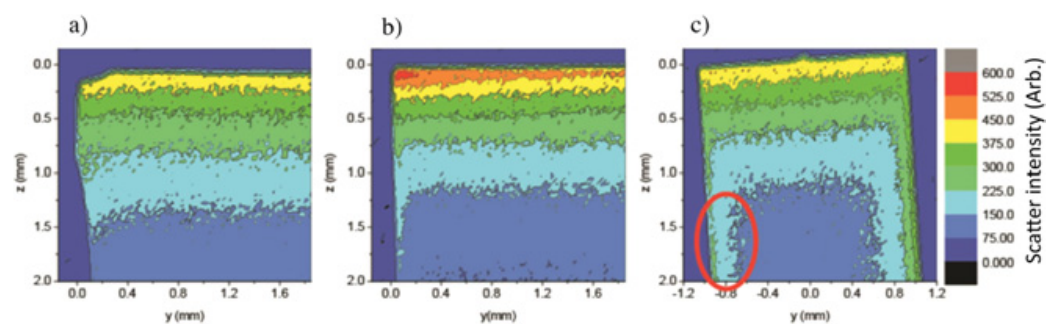

Figure 5. 2D images of aerogels using confocal $\mu \mathrm{XRF}$ of a) a cast aerogel with no release agent, b) a cast aerogel with release agent, and c) an aerogel that was diamond turned, showing higher density regions along the side (circled in red) due to pore collapse caused by the machining tool. Figure is referenced in B. Patterson et.al, X-ray Spectrometry 41, 253 (2012).

Fig. 5(a) and Fig. 5(b) along the upper surface shows a higher density in the aerogel cast with release agent. This data shows that the releasing agent 'wicks' into the aerogel during formation and leaves behind a higher density skin. The diamond turned aerogel in Fig. 5(c) shows higher density regions along the sides (indicated with red oval) due to void collapse due to tool pressure.

\section{CONCLUSIONS}

LANL is currently engaged in several target platforms on NIF, both in collaborative effort as well as experimentation. The collaborative efforts with LANL and GA on the Be-Cu ignition capsules have shown through both $\mu$-CT at LANL and contact radiography at GA that $\mathrm{Cu}$ migrates radially from the inside of the capsule towards the capsule surface forming $\mathrm{Cu}$ domes along the surface [2, 3]. This is invaluable data for manufacturing of sputtered $\mathrm{Be}-\mathrm{Cu}$ capsules. In addition, LANL is also engaged in target manufacturing NIF targets requiring aerogel. One important characterization metric the physics' has determined is that the knowledge of density gradients in foams is important. We have located these density gradients in aerogels and foams using $3 \mathrm{D}$ confocal micro $\mathrm{x}$-ray fluorescence $(\mu \mathrm{XRF})$ imaging and 3D x-ray computed tomography (CT) imaging and have shown that the gradients are formed as a result of how they are manufactured.

\section{References}

[1] D.B. Butrymowicz, J.R. Manning, M.E. Read, J. Phys. Chem. Ref. Data 2, 643 (1973)

[2] B.M. Patterson, K.A. DeFriend Obrey, G.J. Havrilla, A. Nikroo, H. Huang, Fusion Science and Technology 55, 4 (2009)

[3] B.M. Patterson, K.A. Obrey, G.J. Havrilla, Fusion Science and Technology 59, 1 (2011)

[4] K.A. DeFriend Obrey, R.D. Day, D. Hatch, B.F. Espinoza, S. Feng, B.M. Patterson, Fusion Science and Technology 55, 4 (2009)

[5] C.E. Hamilton, D. Honnell, B.M. Patterson, D.W. Schmidt, K.A. DeFriend Obrey, Fusion Science and Technology 59, 1 (2011)

[6] B.M. Patterson, K.A.D. Obrey, C.E. Hamilton, G.J. Havrilla, X-ray Spectrometry 41, 253 (2012) 